\title{
Is there a place for a single value oral glucose tolerance test for the diagnosis of gestational diabetes mellitus?
}

(Index words: Fasting plasma glucose, 2-h plasma glucose, WHO criteria)

The oral glucose tolerance test (OGTT) is the recommended diagnostic test for gestational diabetes mellitus (GDM). There is no consensus regarding the diagnostic criteria and OGTTs described by the World Health Organization (WHO) [1] and the American Diabetes Association [2] are the tests most frequently used.

In 1999, the WHO changed the threshold values for the $75 \mathrm{~g}$ OGTT to a fasting venous plasma glucose (FPG) value of $\geq 7.0 \mathrm{mmol} / \mathrm{L}$ and/or a 2 -h value of $\geq 7.8 \mathrm{mmol} / \mathrm{L}$ [1]. One reason for this amendment was a discrepancy in the sensitivity of the previous threshold values [3]. The WHO criteria are based on studies done on non-pregnant populations, which show an increase in long term complications above these values [3].

South Asians are a high risk group for developing GDM and it is recommended that they be screened routinely [2]. In our unit, we screen only those with a further risk factor, such as obesity or a positive family history. We reviewed 2500 consecutive OGTTs performed in our Reproductive Biology Laboratory on pregnant women from January 2002 to July 2003. An automated glucose oxidase colorimetric test kit was used to assay venous plasma glucose levels.

Five hundred and twenty two (20.9\%) had GDM by the current WHO criteria. The abnormalities in the GTTs are given in Table 1. The data show that if the OGTT was performed with only the 2-h value, $99.6 \%$ of the women with GDM would have been diagnosed with only a minimal loss of sensitivity.

Table 1. Oral glucose tolerance test (OGTT) results of women with gestational diabetes mellitus $(n=522)$

\begin{tabular}{lcc}
\hline Abnormality on OGTT & $\begin{array}{c}\text { Number of } \\
\text { diabetics (\%) }\end{array}$ & $95 \%$ CI \\
\hline $\begin{array}{l}\text { Elevated FPG and } \\
\text { 2-h values }\end{array}$ & $480(92.0)$ & $90.8-93.2$ \\
$\begin{array}{l}\text { Elevated 2-h value } \\
\text { with normal FPG }\end{array}$ & $40(7.6)$ & $6.4-8.8$ \\
$\begin{array}{l}\text { Elevated FPG with normal } \\
\text { 2-h value }\end{array}$ & $2(0.4)$ & $0.1-0.7$ \\
Total & $\mathbf{5 2 2 ( 1 0 0 )}$ & \\
\hline
\end{tabular}

FPG-fasting plasma glucose
Such an approach would be useful in poorer regions such as South Asia, where the burden of disease is high and the resources are limited. There are an estimated 35.5 million diabetics in India alone [4]. A single-value GTT would make screening for GDM more efficient and less cumbersome for the mother and the health care system. Further, in many low resource settings, glucose estimations are done manually. This is a time consuming and labour intensive method compared to the automated techniques available in more sophisticated laboratories.

An oral glucose tolerance test using only the 2-h plasma glucose would be a cost effective way of diagnosing GDM.

\section{References}

1. World Health Organization. Definition, diagnosis, and classification of diabetes mellitus and its complications. Geneva: World Health Organization, 1999.

2. American Diabetes Association. Position Statement 2003: Gestational diabetes mellitus. Diabetes Care 2003; 26: S103-5.

3. The Expert Committee on the Diagnosis and Classification of Diabetes Mellitus. Follow-up report on the diagnosis of diabetes mellitus. Diabetes Care 2003; 26: 3160-7.

4. International Diabetes Federation. Diabetes Atlas. 2nd ed., 2003. [http://www.idf.org]

Hemantha Senanayake, Senior Lecturer, Hemal Ariyaratne, Research Assistant and Sumedha Wijeratne, Lecturer, Department of Obstetrics and Gynaecology, Faculty of Medicine, University of Colombo, Sri Lanka.

Correspondence: HS, e-mail: <hsenan@sltnet.lk> (Competing interests: none declared). Received 18 June 2004 and accepted 7 August 2004. 